\title{
One hundred years of Lewis Chemical Bond!
}

MS received 19 September 2016; accepted 20 September 2016

When a suggestion about a special issue on the Chemical Bond came to us from the Editor, we readily agreed. After nine months, we are pleased to present the special issue of the Journal of Chemical Science to celebrate the $100^{\text {th }}$ year of the 'electron pair bond' proposed by G. N. Lewis. ${ }^{1}$ By any stretch of imagination of that period, this was a conceptual quantum jump, coming more than a decade ahead of quantum mechanics and the Schrödinger's equation. However, the periodic table of elements was already available. Chemists knew how many electrons are there in each element and were also aware of stable electronic configurations. For example, 'inert gases' having 8 electrons in the valence shell (now known as $s$ and $p$ orbitals) were very stable. Bonding in polar molecules, called electrovalent those days, such as $\mathrm{KCl}$ could be understood based on Coulombic interaction between the oppositely charged $\mathrm{K}^{+}$and $\mathrm{Cl}^{-}$. It was a puzzle for chemists to understand how neutral molecules such as $\mathrm{H}_{2}, \mathrm{~F}_{2}$ and $\mathrm{HF}$ are bound! In the 'electrovalent molecules', an electron is transferred from one atom $(\mathrm{K})$ to another $(\mathrm{Cl})$, forming respectively $\mathrm{K}^{+}$and $\mathrm{Cl}^{-}$.

Having understood the stable electronic configuration of the rare gases, Lewis suggested that $\mathrm{H}$ and $\mathrm{F}$ atom can both attain such configuration, if they were to share one electron with each other. He developed a cubic model of atoms with each surrounded by 8 electrons after forming electron pair bonds. He put a 'colon' in between the two atoms, such as H:F, the two dots representing two electrons. Now, $\mathrm{H}$ could have two electrons resembling the stable configuration of $\mathrm{He}$ and $\mathrm{F}$ could have eight electrons resembling the stable configuration of Ne. Though Lewis did use a 'cubic model of atoms' with eight electrons in their corners, he realized that electrons pairing is more fundamental than the magic number of eight. In his own words: The rule of eight, in spite of its great importance, is less fundamental than the rule of two, which calls attention to the tendency of electrons to form pairs. ${ }^{2}$ Lewis called it 'electron pair bonding'. Langmuir extended this work with some of his own ideas and gave it a name that became widely used: covalent bond. ${ }^{3}$ Interestingly, the name 'covalent bond' survived replacing 'electron pair bond' from the literature. Electrovalent bond became ionic bond eventually. Langmuir also emphasized the rule of eight and called it 'octet rule', about which Lewis was not convinced at all. He was not amused when others started referring to these advances as Lewis-Langmuir theory. In his own words: ${ }^{2}$ the theory has been designated in some quarters as the Lewis-Langmuir theory, which would imply some sort of collaboration (when there was none). They were both giants in Chemistry and life had different plans for these two great chemists. Lewis wanted his discoveries to be credited to him and whatever extension Langmuir did to be credited to Langmuir. We refer to an article by Jensen in the Journal of Chemical Education for a historical account. ${ }^{4}$ Respecting Lewis's views, this special issue is called ' 100 years of Lewis Chemical Bond'.

While it might sound trivial today, the suggestion that two electrons, both having a negative charge come together to form a bond between two atoms is dramatic. Of course, the intuitive feeling coming from the knowledge of chemistry thus far about the electron pair bond was so strong that, Lewis was even willing to speculate that the electrostatic repulsion between like charges at very short distance may not follow the usual rules. The existence of multi-electron atoms should have given him the confidence to propose this model. This, still, required much courage as the theories of bonding, MO and VB, based on quantum mechanics, and properties of spin of electrons were yet to appear. While it is all understood from the quantum physics very well today, the 'chemical bond' still appears mysterious to many.

Adding to the mystique of chemical bonds is intermolecular bonding that is exploding in chemistry literature over the last decade or so. Not surprisingly, these developments are not as novel as one might think. Within 4 years after the Lewis's 'electron pair bond', Latimer and Rodebush from the same department in the University 
of California, Berkeley, came up with the suggestion of a 'hydrogen bond'. 5 They set out to explain the unique nature of $\mathrm{H}_{2} \mathrm{O}$ in comparison to $\mathrm{HCl}$ and $\mathrm{NH}_{3}$. They pointed out that $\mathrm{H}_{2} \mathrm{O}$ can give up or accept a proton leading to $\mathrm{OH}^{-}$or $\mathrm{H}_{3} \mathrm{O}^{+}$readily but $\mathrm{NH}_{3}$ prefers to accept a proton more readily leading to $\mathrm{NH}_{4}^{+}$, and $\mathrm{HCl}$ prefers to give up a proton more readily leading to $\mathrm{Cl}^{-}$. They suggested that this happens because $\mathrm{H}$ from one $\mathrm{H}_{2} \mathrm{O}$ molecule could have attractive interaction with the lone pair of electrons in the $\mathrm{O}$ from a neighbouring molecule. As it would have violated the 'octet rule' of Lewis, they concluded that a $\mathrm{H}$ held between two octets constitutes a 'weak bond', making amends with the octet rule. We know the hydrogen bond better now and have learned that both $\mathrm{NH}_{3}$ and $\mathrm{HCl}$ and also $\mathrm{CH}_{4}$ and $\mathrm{H}_{2} \mathrm{~S}$ can all accept and donate hydrogen bonds. We have learned that hydrogen is not unique in bringing two molecules closer, whether in isolated complexes or in a crystal. Every element in the periodic table could in principle have similar 'bonds'. However, the influence of Lewis has been phenomenal and 'chemical bond' has become synonymous with 'covalent bond' for most of the chemists, in a way that hinders the progress in this field. Once again quantum mechanics is beginning to bridge the gap, to see a continuum in chemical bonding.

This issue has articles on all types of bonds, not limited to the 'covalent bond' proposed by Lewis and named so by Langmuir. It covers the bond between noble gases and noble metals in an article by Ghara, Pan, Deb, Kumar, Sarkar and Chattaraj. Though the inertness of the 'noble gases' led Lewis to propose the covalent bond, it did not take long for chemists to realize that noble gases can form bonds with other elements, under appropriate conditions. Hydrogen bonding is discussed in articles by Banerjee, Bhattacharya and Chakraborty; Karir, Fatima and Viswanathan; and Shahi and Arunan. Pavan and Guru Row discuss $\mathrm{Cl} \cdots \mathrm{Cl}$ and $\mathrm{Cl} \cdots \mathrm{Br}$ halogen bonds in the crystal structure of 4-bromo-2-chlorobenzoic acid. Shukla and Chopra discuss the substitution effect on S. . F chalcogen bond. Dhindhwal and Sathyamurthy describe the effect of hydration on cation- $\pi$ interactions between benzene and various cations. Coordinate bonding is discussed in articles by Kathuria, Arfeen, Bankar and Bhartam, and Gupta Velmurugan, Rajeshkumar and Rajaraman. Bonding properties on high energy solids under high pressure is discussed in the article by Vaitheeswaran, Yedukondalu and Abraham. Lithium adsorption on monolayers of carbonaceous materials is discussed by Panigrahi, Umadevi and Narahari Sastry. Choudhuri, Mahata, Rawat and Pathak discuss the effect of Ti doping in $\mathrm{Al}\left(\mathrm{BH}_{4}\right)_{3}$ on the dehydrogenation reactions. Jemmis and Priyakumari discuss bonding in hypervalent oligo-sulfuranes. Sasmal, Talukdar, Nayak, Vaval and Pal discuss the hyperfine structure constants in small molecules and these reflect the nature of bonding in these molecules. Gadre and Kumar; Bijina and Suresh; and Vinod Kumar, Raghavendra and Subramanian discuss the importance of electrostatic potential/electron density topology in understanding various types of bonding.

The articles in this special issue demonstrate the variety in bonding and the many ways, both experimental and theoretical, of investigating them. We thank the enthusiastic support received from all the authors, who have contributed to this special issue. We also thank the Editor for the invitation and support and the members of the Journal staff for the excellent support during the compilation of this issue.

\section{References}

1. Lewis G N 1916 J. Am. Chem. Soc. 38762

2. Lewis G N 1923 In Valence and the Structure of Atoms and Molecules (New York: Chemical Catalog Co.)

3. Langmuir I $1919 \mathrm{~J}$. Am. Chem. Soc. 41868

4. Jensen W B 1984 J. Chem. Educ. 61191

5. Latimer W M and Rodebush W H 1920 J. Am. Chem. Soc. 421419

E ARUNAN $^{\mathrm{a}}$, E D JEMMIS ${ }^{\mathrm{a}}$ and V SUBRAMANIAN ${ }^{\mathrm{b}}$ ${ }^{a}$ Department of Inorganic and Physical Chemistry, Indian Institute of Science, Bangalore 560 012, India ${ }^{\mathrm{b}}$ Chemical Laboratory, CSIR-Central Leather Research Institute, Adyar, Chennai 600 020, India e-mail: arunan@ipc.iisc.ernet.in; jemmis@ipc.iisc.ernet.in; subbu@clri.res.in 